Journal of Mathematics and Statistics 7 (3): 249-254, 2011

ISSN 1549-3644

(C) 2011 Science Publications

\title{
A New Perturbative Approach in Nonlinear Singularity Analysis
}

\author{
Tat-Leung Yee \\ Department of Mathematics and Information Technology, \\ The Hong Kong Institute of Education, Tai Po, \\ New Territories, Hong Kong
}

\begin{abstract}
Problem statement: The study is devoted to the "mirror" method which enables one to study the integrability of nonlinear differential equations. Approach: A perturbative extension of the mirror method is introduced. Results: The mirror system and its first perturbation are then utilized to gain insights into certain nonlinear equations possessing negative Fuchs indices, which were poorly understood in the literatures. Conclusion/Recommendations: In particular, for a nonprincipal but maximal Painleve family the first-order perturbed series solution is already a local representation of the general solution, whose convergence can also be proved.
\end{abstract}

Key words: Mirror transformation, painleve test, singularity analysis, Ordinary Differential Equations (ODE), singularity analysis, mirror system, maximal family, perturbation expansion, negative Fuchs indices

\section{INTRODUCTION}

The relevant literature study dates back to one century ago when Painleve made an in-depth study of singularities and initiated the (now named) Painleve analysis of integrability. Painleve set up the problem of determining all differential equations whose general solution are single-valued. Following the pioneering work of Painleve 1902, the methods of Gambier 1909 Bureau (1964); Ablowitz et al. (1980) and Weiss et al. (1983) have been evolved and they were successful to apply in many cases. However, the main drawback of the methods is that none of them can build necessary conditions at all integer values of "resonances". To be specific, negative Fuchs indices cannot be handled by these methods. The reason why the methods cannot handle negative indices lies in the fact that their Laurent series is assumed to be bounded from below.

The mirror method uses the new tool in singularity analysis: mirror transformations and regular mirror systems, which was first introduced by $\mathrm{Hu}$ and Yan $(1999 ; 2000)$. By this method they were successful in the following several aspects. Firstly, the success of constructing mirror transformations enables us to treat each principal balance in the Painleve test, singularity structures and symplectic structures of Hamiltonian systems from a common point of view. Secondly, Hu et al. (2001) showed that the mirror transformations are canonical for finite-dimensional Hamiltonian systems. Moreover, Yee (2002) demonstrated that the linearization of mirror systems near movable poles gives the possibility to construct the associated Backlund transformations of some partial differential equations and the Schlesinger transformations of some ordinary differential equations.

In the current work our primary goal is to introduce an improvement of the mirror method so that negative indices ("resonances") can be treated. The structure of the study can now be explained. The perturbative Painleve method is first introduced. We demand single-valuedness not only for any pole-like expansion as in the Painleve test, but also for every solution close to it, represented as a perturbation series in a small parameter $\varepsilon$. The usage of the idea of the perturbative method proves to be tremendously beneficial for the mirror method as a new improvement. Order-zero is the usual mirror system. Order-one reduces to a linearization of mirror system near a regular singularity and allows the introduction of all missing arbitrary coefficients. Higher orders lead to the analysis of a linear, Fuchsian type inhomogeneous system. In particular, negative indices give rise to doubly infinite Laurent series. An illustrative example of Bureau's equation is also presented and finally the conclusion follows. 


\section{MATERIALS AND METHODS}

Perturbative Painleve analysis: Now we first present the perturbative method originally developed by Conte et al. (1993). The method allows us to extract the information contained in the negative indices, thus building infinitely many necessary conditions for the absence of movable critical singularities of the logarithmic type.

Let us consider a nonlinear ordinary differential equation:

$\mathrm{E} \equiv \mathrm{K}(\mathrm{u}, \mathrm{x})=0$

which is polynomial in $\mathrm{u}$ and its derivatives, analytic in $\mathrm{x}$. The standard Painleve expansion takes the form ( $\mathrm{X}$ is the expansion variable, $X_{x}=1$ ):

$$
\begin{aligned}
& u=u^{(0)} \equiv \sum_{j=0}^{+\infty} u_{j}^{(0)} X^{j+p} \\
& E=E^{(0)} \equiv \sum_{j=0}^{+\infty} E_{j}^{(0)} X^{j+q}=0
\end{aligned}
$$

in which the negative integers $\mathrm{p}$ and $\mathrm{q}$ are the respective singularity order of $u$ and $E$.

We seek a Laurent expansion for any solution which is near to the solution obtained by the standard Painleve method. We do this by considering a perturbation expansion. For a non-principal but maximal Painleve family the perturbation extends the particular solution into a representation of the general solution. Let us define the Painleve expansion $\left(\mathrm{u}^{(0)}, \mathrm{E}^{(0)}\right)$ as the solution of unperturbed problem, and look for a nearby solution formally represented by an infinite perturbation series in powers of small parameter $\varepsilon$ :

$$
\begin{aligned}
& \mathrm{u}=\text { Taylor }(\varepsilon) \equiv \sum_{\mathrm{n}=0}^{+\infty} \varepsilon^{\mathrm{n}} \mathrm{u}^{(\mathrm{n})} \\
& \mathrm{K}(\mathrm{u}, \mathrm{x}) \equiv \mathrm{E}=\sum_{\mathrm{n}=0}^{+\infty} \varepsilon^{\mathrm{n}} \mathrm{E}^{(\mathrm{n})}=0
\end{aligned}
$$

Let us denote, for the equation $E=0, R_{k}=$ the set of indices for $\mathrm{k}^{\text {th }}$ family $=\{\ldots,-1, \ldots\}$, with the following assumptions: (1) all indices are distinct integers, and $\mathrm{s}$ to be the smallest index $(\mathrm{s} \leq-1)$; (2) all $\mathrm{k}$ families are maximal (families with a number of indices equal to the order of the equation); (3) at least one of the $\mathrm{k}$ families is principal (any maximal family with, apart from -1, all integer indices non-negative).

Now, the condition that the perturbation expansion still be a solution generates an infinite sequence of successive differential equations:
$\mathrm{E}^{(0)}=\mathrm{K}\left(\mathrm{u}^{(0)}, \mathrm{x}\right)=0$,

$\mathrm{E}^{(1)}=\mathrm{K}^{\prime}\left(\mathrm{u}^{(0)}\right) \mathrm{u}^{(1)}=0, \ldots$,

where, $K^{\prime}$ is the Frechet operator acting on $u^{(\mathrm{n})}$. At each level of perturbation, we construct a pole expansion, but the order of the pole increases with the order of the perturbation. The resulting infinite perturbation expansion is a doubly infinite Laurent expansion:

$$
\begin{aligned}
& u=\sum_{n=0}^{+\infty} \varepsilon^{n}\left[\sum_{j=n s}^{+\infty} u_{j}^{(n)} X^{j+p}\right]=\sum_{j=-\infty}^{+\infty} u_{j} X^{j+p} \\
& E=\sum_{n=0}^{+\infty} \varepsilon^{n}\left[\sum_{j=n s}^{+\infty} E_{j}^{(n)} X^{j+q}\right]=\sum_{j=-\infty}^{+\infty} E_{j} X^{j+q}=0
\end{aligned}
$$

In general, perturbation theory practically always yields divergent series. However, by considering the perturbation series solution $\mathrm{u}^{(0)}+\varepsilon \mathrm{u}^{(1)}$ and expanding in $\mathrm{X}$, with coefficients dependent of $\varepsilon$, we can prove the convergence. This can be done by introducing a new transformation for the mirror system. Eventually we succeed to deduce a regular extended mirror system with regular initial data. The Cauchy-Kowalevski theorem is then applied and convergence follows accordingly. The importance of the perturbed solution $\mathrm{u}^{(0)}+\varepsilon \mathrm{u}^{(1)}$ is that: for a non-principal but maximal Painleve family it is already a local representation of the general solution.

In the following we investigate in detail each order of $\varepsilon$ :

With $\mathrm{n}=0, \mathrm{E}^{(0)}\left(\mathrm{u}^{(0)}\right) \equiv \mathrm{K}\left(\mathrm{u}^{(0)}, \mathrm{x}\right)=0$

where, $u^{(0)}$ is a (either particular or general) solution of the original nonlinear equation, which is determined by standard Painleve analysis:

$=\mathrm{X}^{\mathrm{p}}\left(\mathrm{A}_{0}+\mathrm{B}_{0} \mathrm{X}+\ldots\right), \quad \mathrm{A}_{0} \neq 0$

With $\mathrm{n}=1, \mathrm{E}^{(1)}\left(\mathrm{u}^{(0)}, \mathrm{u}^{(1)}\right) \equiv \mathrm{K}^{\prime}\left(\mathrm{u}^{(0)}\right) \mathrm{u}^{(1)}=0$

where, $u^{(1)}$ is (the general solution of homo equation) + (a particular solution of inhomo equation):

$=X^{\mathrm{p}}\left(\mathrm{A}_{1} \mathrm{X}^{\mathrm{s}}+\mathrm{B}_{1} \mathrm{X}^{\mathrm{s}-1}+\ldots\right)+0$

where, $\mathrm{A}_{1}, \mathrm{~B}_{1}, \ldots$ are arbitrary coefficients introduced at level one. The Painleve series $\mathrm{u}^{(0)}$, a Laurent series which is bounded below, is substituted into the linearized equation $\mathrm{K}^{\prime}\left(\mathrm{u}^{(0)}\right) \mathrm{u}^{(1)}=0$, the resulting equation for $\mathrm{u}^{(1)}$ is of Fuchsian type, the movable 
singularity $\mathrm{X}=0$ of the original ODE is a regular singularity for the linearized equation and its Fuchs indices are $i+p$, where $i$ runs over the Painleve resonances.

At this first order, an arbitrary coefficient is introduced at each index. Not all of these are new since we already have a coefficient in $\mathrm{u}^{(0)}$, corresponding to each positive integer index. The coefficients introduced into $\mathrm{u}^{(1)}$ at the corresponding indices $(\mathrm{i}+\mathrm{p}, \mathrm{i}$ a positive integer) just perturb the already arbitrary coefficients, so add nothing new and it is not harmful to set them zero at this level. However, all other indices give rise to new arbitrary coefficients. Therefore the expression $u^{(0)}+\varepsilon u^{(1)}$ already contains as many arbitrary coefficients as there are indices in the family:

With $n \geq 2, E^{(n)}\left(u^{(0)}, \ldots, u^{(n)}\right) \equiv K^{\prime}\left(u^{(0)}\right) u^{(n)}-R^{(n)}\left(u^{(0)}\right.$, $\left.\ldots, \mathrm{u}^{(\mathrm{n}-1)}\right)=0$

Where:

$\begin{aligned} u^{(n)} & =X^{p}\left(A_{n} X^{s}+B_{n} X^{s-1}+\ldots\right) \\ & +X^{p}\left(C_{n} X^{s n}+D_{n} X^{s n-1}+\ldots\right)\end{aligned}$

where, $A_{n}, B_{n}, \ldots$ are arbitrary (independent of $A_{i}, B_{i}, i$ $\leq \mathrm{n}$-1) $\mathrm{n}$-th level coefficients that can be absorbed by $u^{(1)}$. Without the loss of generality, we set $A_{n}=B_{n}=0$ for each $n \geq 2$. Therefore, for $n \geq 2$, we only concern about a particular solution of each inhomogeneous equation. The coefficients $C_{n}, D_{n}, \ldots$ are dependent of the previous "useful" coefficients which belong to a subset of $\left\{A_{i}, B_{i}, \ldots \mid i=0,1\right\}$.

At these $n$-th orders $(n \geq 2)$ each function $u^{(n)}$ satisfies an inhomogeneous, linear differential equation. The indicial equation is the same for all $\mathrm{n} \geq 1$ but for $\mathrm{n}$ $\geq 2$ the leading behaviour of $u^{(n)}$ is determined by the singularity order of the rhs function $\mathrm{R}^{(\mathrm{n})}$, not by $\mathrm{K}^{\prime}\left(\mathrm{u}^{(0)}\right)$.

\section{RESULTS}

We begin to illustrate, through a simple but instructive example, that the analysis on mirror systems might be performed in a perturbative approach such that negative and positive indices can be treated at the same time. We illustrate the algorithm of performing the new perturbative approach on the mirror system through the following ODE example of third-order kind, namely the Bureau's equation. We also aim at showing the proof of convergence of the no principal balance of mirror system. where:

The Bureau's third-order ODE is $\mathrm{E}=\mathrm{K}(\mathrm{u}, \mathrm{t})=0$,

$\mathrm{K}(\mathrm{u}, \mathrm{t}) \equiv \mathrm{u}^{\prime \prime \prime}+3 \mathrm{uu} \mathrm{u}^{\prime \prime}+3\left(\mathrm{u}^{\prime}\right)^{2}+\left(3 \mathrm{u}^{2}-\mathrm{c}_{0}\right) \mathrm{u}^{\prime}-\mathrm{c}_{0}{ }^{\prime} \mathrm{u}-\mathrm{d}_{0}$,

where, $c_{0}$ and $d_{0}$ are functions of t. By the standard Painleve test we obtain two families of solutions with singularity orders and Fuchs indices in the following:

(F1) $\mathrm{p}=-1, \mathrm{u}_{0}=1,\{-1,1,3\}$,

(F2) $\mathrm{p}=-1, \mathrm{u}_{0}=2,\{-2,-1,3\}$

The Painleve series of (F1)-(F2) are respectively:

$\mathrm{u}^{(0)}=\mathrm{T}^{-1}+\mathrm{r}_{2}+\left(-\mathrm{r}_{2}^{2}+\frac{\mathrm{c}_{0}\left(\mathrm{t}_{0}\right)}{3}\right) \mathrm{T}+\mathrm{r}_{3} \mathrm{~T}^{2}+\ldots$

And:

$\mathrm{u}^{(0)}=2 \mathrm{~T}^{-1}+\frac{\mathrm{c}_{0}\left(\mathrm{t}_{0}\right)}{6} \mathrm{~T}+\mathrm{s}_{2} \mathrm{~T}^{2}$

$+\left(-\frac{\mathrm{c}_{0}^{2}\left(\mathrm{t}_{0}\right)}{360}+\frac{\mathrm{d}_{0}\left(\mathrm{t}_{0}\right)}{30}-\frac{\mathrm{c}_{0}{ }^{\prime \prime}\left(\mathrm{t}_{0}\right)}{30}\right) \mathrm{T}^{3}+\ldots$

where, $\mathrm{T}:=\mathrm{t}-\mathrm{t}_{0}$ and $\mathrm{t}_{0}, \mathrm{r}_{2}, \mathrm{r}_{3}, \mathrm{~s}_{2}$ are arbitrary.

Now we are applying the perturbative Painleve analysis to the second family (F2) and the result reads:

$\mathrm{u}^{(0)}=\mathrm{T}^{-1}\left[2+\frac{1}{6} \mathrm{c}_{0}\left(\mathrm{t}_{0}\right) \mathrm{T}^{2}+\mathrm{s}_{2} \mathrm{~T}^{3}+\ldots\right]$,
$\mathrm{u}^{(1)}=\mathrm{T}^{-1}\left[\mathrm{~A}_{1} \mathrm{~T}^{-2}+0 \mathrm{~T}^{-1}+\mathrm{A}_{1}\left(\frac{1}{6} \mathrm{c}_{0}\left(\mathrm{t}_{0}\right)-\frac{3}{2} \mathrm{~S}_{2}\right) \mathrm{T}\right.$
$\left.+\mathrm{A}_{1}\left(-\frac{1}{240} \mathrm{c}_{0}^{2}\left(\mathrm{t}_{0}\right)-\frac{1}{30} \mathrm{~d}_{0}\left(\mathrm{t}_{0}\right)+\frac{1}{120} \mathrm{c}_{0}{ }^{\prime}\left(\mathrm{t}_{0}\right)\right) \mathrm{T}^{2}+0 \mathrm{~T}^{3}+\ldots\right]$

Thus, the resulting infinite perturbation expansion for (F2) is a doubly infinite Laurent expansion:

$u=u^{(0)}+\varepsilon u^{(1)}+\varepsilon^{2} u^{(2)}+\ldots+\varepsilon^{n} u^{(n)}+\ldots=T^{-1} \sum_{j=-\infty}^{+\infty} u_{j} T^{j}$

The local representation of the general solution is given by:

$\mathrm{u}^{*}=\mathrm{u}^{(0)}+\varepsilon \mathrm{u}^{(1)}=\left(\varepsilon \mathrm{A}_{1}\right) \mathrm{T}^{-3}+2 \mathrm{~T}^{-1}$

$+\varepsilon A_{1}\left(\frac{1}{6} c_{0}\left(t_{0}\right)-\frac{3}{2} \mathrm{~s}_{2}\right) \mathrm{T}^{0}+\ldots$

To demonstrate explicitly the extension of the mirror method we introduce the corresponding mirror transformation for the original equation given by (7):

$\left\{\begin{array}{l}u=\theta^{-1}, \\ v=-\theta^{-2}+\eta_{2} \theta^{-1}, \\ w=2 \theta^{-3}-3 \eta_{2} \theta^{-2}+c_{0} \theta^{-1}+\eta_{3}\end{array}\right.$ 
The regular mirror system is given by:

$$
\left\{\begin{array}{l}
\theta^{\prime}=1-\eta_{2} \theta \\
\eta_{2}{ }^{\prime}=c_{0}-\eta_{2}^{2}+\eta_{3} \theta \\
\eta_{3}{ }^{\prime}=d_{0}
\end{array}\right.
$$

The above mirror system can be expressed as $\mathrm{K}(\Theta)$ $=0$, where $\Theta=\left(\theta, \eta_{2}, \eta_{3}\right)$. Based on the dominant balance we obtain the two families of solutions of the mirror system with the following singularity orders and Fuchs indices:

(F1) $\mathrm{p}=(1,-1,-3), \Theta=(1,0,0),\{-1,1,3\}$,

(F2) $\mathrm{p}=(1,-1,-3), \quad \Theta=\left(\frac{1}{2}, 1,0\right), \quad\{-2,-1,3\}$

The Painleve series of (F1)-(F2) are respectively:

$$
\left\{\begin{array}{l}
\theta^{(0)}=\mathrm{T}\left[1-\mathrm{r}_{2} \mathrm{~T}+\left(2 \mathrm{r}_{2}^{2}-\frac{1}{3} \mathrm{c}_{0}\left(\mathrm{t}_{0}\right)\right) \mathrm{T}^{2}\right. \\
\left.+\left(-3 \mathrm{r}_{2}^{3}-\mathrm{r}_{3}+\frac{2}{3} \mathrm{r}_{2} \mathrm{c}_{0}\left(\mathrm{t}_{0}\right)\right) \mathrm{T}^{3}+\ldots\right], \\
\eta_{2}^{(0)}=\mathrm{T}^{-1}\left[0+2 \mathrm{r}_{2} \mathrm{~T}+\left(-4 \mathrm{r}_{2}^{2}+\mathrm{c}_{0}\left(\mathrm{t}_{0}\right)\right) \mathrm{T}^{2}\right. \\
\left.+\left(4 \mathrm{r}_{2}^{3}+4 \mathrm{r}_{3}-\mathrm{r}_{2} \mathrm{c}_{0}\left(\mathrm{t}_{0}\right)\right) \mathrm{T}^{3}+\ldots\right] \\
\eta_{3}^{(0)}=\mathrm{T}^{-3}\left[0+0 \mathrm{~T}+0 \mathrm{~T}^{2}\right. \\
\left.+\left(-8 \mathrm{r}_{2}^{3}+8 \mathrm{r}_{3}+2 \mathrm{r}_{2} \mathrm{c}_{0}\left(\mathrm{t}_{0}\right)-\mathrm{c}_{0}{ }^{\prime}\left(\mathrm{t}_{0}\right)\right) \mathrm{T}^{3}+\ldots\right]
\end{array}\right.
$$

$$
\left\{\begin{array}{l}
\theta^{(0)}=\mathrm{T}\left[\frac{1}{2}+0 \mathrm{~T}-\frac{1}{24} \mathrm{c}_{0}\left(\mathrm{t}_{0}\right) \mathrm{T}^{2}-\frac{1}{4} \mathrm{~s}_{2} \mathrm{~T}^{3}+\ldots\right] \\
\eta_{2}^{(0)}=\mathrm{T}^{-1}\left[1+0 \mathrm{~T}+\frac{1}{3} \mathrm{c}_{0}\left(\mathrm{t}_{0}\right) \mathrm{T}^{2}+\frac{5}{2} \mathrm{~s}_{2} \mathrm{~T}^{3}+\ldots\right] \\
\eta_{3}^{(0)}=\mathrm{T}^{-3}\left[0+0 \mathrm{~T}+0 \mathrm{~T}^{2}+\left(20 \mathrm{~s}_{2}-2 \mathrm{c}_{0}^{\prime}\left(\mathrm{t}_{0}\right)\right) \mathrm{T}^{3}+\ldots\right]
\end{array}\right.
$$

The perturbative expansion for the mirror system is:

$$
\Theta=\sum_{n=0}^{+\infty} \varepsilon^{\mathrm{n}} \Theta^{(\mathrm{n})}, \Theta^{(\mathrm{n})}=\left(\theta^{(\mathrm{n})}, \eta_{2}^{(0)}, \eta_{3}^{(0)}\right)
$$

$\mathrm{K}(\Theta)=0, \quad \mathrm{~K}\left(\Theta^{(0)}\right)=0$. The first few terms are determined by:

$$
\left\{\begin{array}{l}
\Theta^{(1)}: K^{\prime}\left(\Theta^{(0)}\right) \Theta^{(1)}=0 \\
\Theta^{(2)}: K^{\prime}\left(\Theta^{(0)}\right) \Theta^{(2)}-R^{(2)}\left(\Theta^{(0)}, \Theta^{(1)}\right)=0 \\
\Theta^{(3)}: K^{\prime}\left(\Theta^{(0)}\right) \Theta^{(3)}-R^{(3)}\left(\Theta^{(0)}, \Theta^{(1)}, \Theta^{(2)}\right)=0
\end{array}\right.
$$

Where:

$$
\left\{\begin{array}{l}
\mathrm{K}^{\prime}\left(\Theta^{(1)}\right) \equiv\left(\begin{array}{ccc}
\partial_{\mathrm{t}}+\eta_{2}^{(0)} & \theta^{(0)} & 0 \\
-\eta_{3}^{(0)} & \partial_{\mathrm{t}}+2 \eta_{2}^{(0)} & -\theta^{(0)} \\
0 & 0 & \partial_{\mathrm{t}}
\end{array}\right) \\
\mathrm{R}^{(2)} \equiv\left(\begin{array}{c}
-\theta^{(1)} \eta_{2}^{(1)} \\
-\left(\eta_{2}^{(1)}\right)^{2}+\theta^{(1)} \eta_{3}^{(1)} \\
0
\end{array}\right) \\
\mathrm{R}^{(3)} \equiv\left(\begin{array}{c}
-\theta^{(1)} \eta_{2}^{(2)}-\theta^{(2)} \eta_{2}^{(1)} \\
-2 \eta_{2}^{(1)} \eta_{2}^{(2)}+\theta^{(1)} \eta_{3}^{(2)}+\theta^{(2)} \eta_{3}^{(1)} \\
0
\end{array}\right)
\end{array}\right.
$$

Finally the successive linearizations of mirror system can be determined now. We consider the nonprincipal balance (F2) only. With $n=0$ :

$$
\left\{\begin{array}{l}
\theta_{\mathrm{t}}^{(0)}=1-\eta_{2}^{(0)} \theta^{(0)} \\
\left(\eta_{2}^{(0)}\right)_{\mathrm{t}}=\mathrm{c}_{0}-\left(\eta_{2}^{(0)}\right)^{2}+\eta_{3}^{(0)} \theta^{(0)} \\
\left(\eta_{3}^{(0)}\right)_{\mathrm{t}}=\mathrm{d}_{0}
\end{array}\right.
$$

which determines $\Theta^{(0)}=\left(\theta^{(0)}, \eta_{2}^{(0)}, \eta_{3}^{(0)}\right)$ as given by (16). With $\mathrm{n}=1$ :

$$
\left\{\begin{array}{l}
\theta_{\mathrm{t}}^{(1)}+\eta_{2}^{(0)} \theta^{(1)}+\theta^{(0)} \eta_{2}^{(1)}=0 \\
\left(\eta_{2}^{(1)}\right)_{\mathrm{t}}-\eta_{3}^{(0)} \theta^{(1)}+2 \eta_{2}^{(0)} \eta_{2}^{(1)}-\theta^{(0)} \eta_{3}^{(1)}=0 \\
\left(\eta_{3}^{(1)}\right)_{\mathrm{t}}=0
\end{array}\right.
$$

which gives:

$$
\left\{\begin{array}{l}
\theta^{(1)}=\mathrm{T}\left[\alpha_{1} \mathrm{~T}^{-2}+0 \mathrm{~T}^{-1}-\alpha_{1} \mathrm{c}_{0}\left(\mathrm{t}_{0}\right) \mathrm{T}^{0}+\ldots\right] \\
\eta_{2}^{(1)}=\mathrm{T}^{-1}\left[0 \mathrm{~T}^{-2}+0 \mathrm{~T}^{-1}+0 \mathrm{~T}^{0}+\left(10 \alpha_{1} \mathrm{~s}_{2}-\alpha_{1} \mathrm{c}_{0}{ }^{\prime}\left(\mathrm{t}_{0}\right) \mathrm{T}+\ldots\right]\right. \\
\eta_{3}^{(1)}=\mathrm{T}^{-3}\left[0 \mathrm{~T}^{-2}+0 \mathrm{~T}^{-1}+0 \mathrm{~T}^{0}+0 \mathrm{~T}+\ldots\right]
\end{array}\right.
$$

where $s_{2}$ and $\alpha_{1}$ are independent arbitrary constants introduced at the zero and the first level, which correspond to indices -1 and -2 , respectively. So, $\alpha_{1}$ is the new (important) parameter that we are looking for. At this level, we set another two arbitrary constants to zero without any loss of generality since the arbitrary constants (at indices -1 and 3 ) are already represented into $\Theta^{(0)}$. Since the family (F2) is maximal then the perturbed solution $\Theta^{(0)}+\varepsilon \Theta^{(1)}$ is a local representation of the general solution. One indeed can continue to look for higher level perturbation in order to obtain a doubly infinite expansion. We just list the second level linearization of mirror system below and the information up to $\mathrm{n}=1$ is good enough for our purpose.

With $\mathrm{n}=2$ : 
J. Math. \& Stat., 7 (3): 249-254, 2011

$\left\{\begin{array}{l}\theta_{\mathrm{t}}^{(2)}+\eta_{2}^{(0)} \theta^{(2)}+\theta^{(0)} \eta_{2}^{(2)}=-\theta^{(1)} \eta_{2}^{(1)} \\ \left(\eta_{2}^{(2)}\right)_{\mathrm{t}}-\eta_{3}^{(0)} \theta^{(2)}+2 \eta_{2}^{(0)} \eta_{2}^{(2)}-\theta^{(0)} \eta_{3}^{(2)}= \\ -\left(\eta_{2}^{(1)}\right)^{2}+\theta^{(1)} \eta_{3}^{(1)} \\ \left(\eta_{3}^{(2)}\right)_{\mathrm{t}}=0\end{array}\right.$

\section{DISCUSSION}

The new transformation for the mirror system can be determined based on the above results. In the following, let us also prove the convergence of the perturbation series solution $\Theta^{(0)}+\varepsilon \Theta^{(1)}$ of (F2). Again, we shall use a new transformation to convert the original mirror system into a new regular system of first-order differential equations with regular initial data.

With the family (F2), we deduce the following Laurent series based on (16) and (20):

$$
\Theta \approx \Theta^{(0)}+\varepsilon \Theta^{(1)}=\left(\begin{array}{l}
\alpha_{1} \varepsilon \mathrm{T}^{-1}+\left(\frac{1}{2}-\frac{1}{6} \alpha_{1} \varepsilon c_{0}\left(\mathrm{t}_{0}\right)\right) \mathrm{T} \\
+\alpha_{1} \varepsilon\left(-\frac{5}{2} \mathrm{~s}_{2}+\frac{1}{6} \mathrm{c}_{0}{ }^{\prime}\left(\mathrm{t}_{0}\right)\right) \mathrm{T}^{2}+\ldots \\
\mathrm{T}^{-1}+\alpha_{1} \varepsilon\left(10 \mathrm{~s}_{2}-\mathrm{c}_{0}{ }^{\prime}\left(\mathrm{t}_{0}\right)\right)+\ldots \\
20 \mathrm{~s}_{2}-2 \mathrm{c}_{0}{ }^{\prime}\left(\mathrm{t}_{0}\right)+\ldots
\end{array}\right)
$$

where, $\alpha_{1}$ and $s_{2}$ are the Painleve resonances at Fuchs indices -2 and 3 , respectively. We easily see that the solution blows up when $\mathrm{T} \rightarrow 0$, or $\mathrm{t} \rightarrow \mathrm{t}_{0}$. We observe that fact that $\eta_{2}$ is the only resonance variable blowing up in the order of $\mathrm{O}\left(\frac{1}{\mathrm{t}-\mathrm{t}_{0}}\right)$. So we first introduce the new variable $\delta$ by $\eta_{2}^{-1}$ and formally invert $\left(t-t_{0}\right)$ into a series of $\delta$. In this example, it is:

$$
\begin{aligned}
& \mathrm{t}-\mathrm{t}_{0}=\delta+\alpha_{1} \varepsilon\left(10 \mathrm{~s}_{2}-\mathrm{c}_{0}{ }^{\prime}\left(\mathrm{t}_{0}\right)\right) \delta^{2} \\
& +\left(\frac{1}{3} \mathrm{c}_{0}\left(\mathrm{t}_{0}\right)+\alpha_{1}^{2} \varepsilon^{2}\left(\mathrm{c}_{0}{ }^{\prime}\left(\mathrm{t}_{0}\right)-10 \mathrm{~s}_{2}\right)^{2}\right) \delta^{3}+\mathrm{O}\left(\delta^{4}\right)
\end{aligned}
$$

Next, we formally expand $\theta$ and $\eta_{3}$ into series of $\delta$ :

$$
\left\{\begin{array}{l}
\theta=\alpha_{1} \varepsilon \delta^{-1}+\alpha_{1}^{2} \varepsilon^{2}\left(\mathrm{c}_{0}{ }^{\prime}\left(\mathrm{t}_{0}\right)-10 \mathrm{~s}_{2}\right) \\
+\frac{1}{2}\left(1-\alpha_{1} \varepsilon \mathrm{c}_{0}\left(\mathrm{t}_{0}\right)\right) \delta+\ldots \\
\eta_{3}=20 \mathrm{~s}_{2}-2 \mathrm{c}_{0}{ }^{\prime}\left(\mathrm{t}_{0}\right)+\ldots
\end{array}\right.
$$

By truncating the $\delta$-series for $\theta$ at the location of the first resonance $\alpha_{1}$ to introduce a new variable $\xi_{2}$, and similarly, truncating the $\delta$-series for $\eta_{3}$ at the location of $s_{2}$ to introduce $\xi_{3}$ we then deduce the new transformation $\left(\theta, \eta_{2}, \eta_{3}\right) \leftrightarrow\left(\delta, \xi_{2}, \xi_{3}\right)$ :

$\theta=\xi_{2} \delta^{-1}, \eta_{2}=\delta^{-1}, \eta_{3}=\xi_{3}$
The extended mirror system becomes:

$$
\left\{\begin{array}{l}
\delta^{\prime}=1-\xi_{2} \xi_{3} \delta-c_{0} \delta^{2} \\
\xi_{2}^{\prime}=-\xi_{2}^{2} \xi_{3}+\left(1-c_{0} \xi_{2}\right) \delta \\
\xi_{3}^{\prime}=d_{0}
\end{array}\right.
$$

The Laurent series for $\left(\delta, \xi_{2}, \xi_{3}\right)$ are:

$$
\left\{\begin{array}{l}
\delta=\mathrm{T}+\alpha_{1} \varepsilon\left(\mathrm{c}_{0}{ }^{\prime}\left(\mathrm{t}_{0}\right)-10 \mathrm{~s}_{2}\right) \mathrm{T}^{2}+\ldots, \\
\xi_{2}=\alpha_{1} \varepsilon+\alpha_{1}^{2} \varepsilon^{2}\left(\mathrm{c}_{0}{ }^{\prime}\left(\mathrm{t}_{0}\right)-10 \mathrm{~s}_{2}\right) \mathrm{T}+\ldots, \\
\xi_{3}=20 \mathrm{~s}_{2}-2 \mathrm{c}_{0}{ }^{\prime}\left(\mathrm{t}_{0}\right)+\ldots
\end{array}\right.
$$

which gives the initial data:

$$
\left(\delta, \xi_{2}, \xi_{3}\right)\left(\mathrm{t}_{0}\right)=\left(0, \alpha_{1} \varepsilon, 20 \mathrm{~s}_{2}-2 \mathrm{c}_{0}{ }^{\prime}\left(\mathrm{t}_{0}\right)\right)
$$

The convergence of the general solution can now be discussed. For a non-principal but maximal family (F2) the first-order perturbed series solution (12) is already a local representation of the general solution. In order to show the convergence of (12), we need the transformations:

$\left(\mathrm{u}, \mathrm{u}^{\prime}, \mathrm{u}^{\prime \prime}\right) \leftrightarrow\left(\theta, \eta_{2}, \eta_{3}\right) \leftrightarrow\left(\delta, \xi_{2}, \xi_{3}\right)$

By the Cauchy-Kowalevski theorem, the extended mirror system (26) with the initial data (28) has a unique analytic solution $\left(\delta(\mathrm{t}), \xi_{2}(\mathrm{t}), \xi_{3}(\mathrm{t})\right)$ near $\mathrm{t}=\mathrm{t}_{0}$.

Then $\left(\theta, \eta_{2}, \eta_{3}\right)=\left(\xi_{2} \delta^{-1}, \delta^{-1}, \xi_{3}\right)$ is a solution of the original mirror system (14) near $\mathrm{t}=\mathrm{t}_{0}$. Moreover, from the ordinary power series method, we can find the expansions for $\left(\delta, \xi_{2}, \xi_{3}\right)$. Then an easy calculation reveals that the Laurent series of $\left(\theta, \eta_{2}, \eta_{3}\right)=\left(\xi_{2} \delta^{-1}, \delta^{-1}\right.$, $\left.\xi_{3}\right)$ are exactly (22). The convergent power series solutions of the extended mirror system lead to convergent Laurent series solutions of the original mirror system, because of the equivalence between the systems. This proves the convergence of $\Theta^{(0)}+\varepsilon \Theta^{(1)}$ in (23). In particular, the series of $\theta^{(0)}+\varepsilon \theta^{(1)}$ is convergent.

From:

$u^{(0)}+\varepsilon u^{(1)}+\ldots=\left(\theta^{(0)}+\varepsilon \theta^{(1)}+\ldots\right)^{-1}$

Or:

$$
\left(\mathrm{u}^{(0)} \boldsymbol{\theta}^{(0)}-1\right)+\varepsilon\left(\mathbf{u}^{(0)} \boldsymbol{\theta}^{(1)}+\mathbf{u}^{(1)} \boldsymbol{\theta}^{(0)}\right)
$$$$
+\varepsilon^{2}\left(u^{(1)} \theta^{(1)}+\ldots\right)+\ldots=0
$$ 
we can find the expansions for $\left(\mathrm{u}^{(0)}, \mathrm{u}^{(1)}\right)$ and they are exactly (12), up to the order where all the resonances appear. This proves the convergence of Laurent series solution $u^{(0)}+\varepsilon u^{(1)}$, which is locally representing the general solution for $(\mathrm{F} 2)$.

\section{CONCLUSION}

In this study we are trying to introduce a patch to the mirror method so that the negative Fuchs indices can be treated. This consideration extends the use of mirror transformations to a larger class of differential equations. Based on the examples under consideration, we are successful in treating the negative Fuchs indices. Order-zero perturbation gives the ordinary mirror system. Order-one reduces to a linearization of mirror system near a regular singularity and allows the introduction of all missing arbitrary coefficients. The method reveals that $u^{(0)}+\varepsilon u^{(1)}$ is already a representation of the general solution, whose convergence can also be proved.

\section{REFERENCES}

Ablowitz, M.J., A. Ramani and H. Segur, 1980. A connection between nonlinear evolution equations and ordinary differential equations of P-type. J. Math. Phys. 21:715-721. DOI: 10.1063/1.524491
Bureau, F.J., 1964. Differential Equations with fixed critical points, Annal. Mat. Pura Appli., 64: 229364. DOI: $10.1007 / \mathrm{BF} 02410054$

Conte, R., A. P. Fordy and A. Pickering, 1993. A perturbative Painleve approach to nonlinear differential equations. Physica D, 69: 33-58. DOI: 10.1016/0167-2789(93)90179-5

Hu, J. and M. Yan, 1999. Singularity analysis for integrable systems by their mirrors. Nonlinearity, 12: 1531-1543. DOI: 10.1088/0951-7715/12/6/306

$\mathrm{Hu}$, J. and M. Yan, 2000. The mirror systems of integrable equations. Stud. Appl. Math., 104: 6790. DOI: $10.1111 / 1467-9590.00131$

Hu, J., M. Yan and T. L. Yee, 2001. Mirror transformations of Hamiltonian systems. Phys. D: Nonlinear Phenomena, 152: 110-123. DOI: 10.1016/S0167-2789(01)00164-6

Weiss, J. M. Tabor and G. Carnevale, 1983. The Painleve property for partial differential equations, J. Math. Phys., 24: 522-526. DOI: $10.1063 / 1.525721$

Yee, T.L., 2002. Linearization of mirror systems. J. Nonlinea. Math. Phys., 9: 234-242. http://citeseerx.ist.psu.edu/viewdoc/download?doi $=10.1 \cdot 1.64 .7539 \& \mathrm{rep}=\mathrm{rep} 1 \&$ type $=$ pdf 